\title{
TTR
}

Traduction, terminologie, re?daction

\section{Le québécisme nordicité : de la néologie à la lexicalisation}

\section{Louis-Edmond Hamelin}

Volume 8, numéro 2, 2e semestre 1995

Technolectes et dictionnaires

URI : https://id.erudit.org/iderudit/037217ar

DOI : https://doi.org/10.7202/037217ar

Aller au sommaire du numéro

Éditeur(s)

Association canadienne de traductologie

ISSN

0835-8443 (imprimé)

1708-2188 (numérique)

Découvrir la revue

Citer cet article

Hamelin, L.-E. (1995). Le québécisme nordicité : de la néologie à la

lexicalisation. TTR, 8(2), 51-65. https://doi.org/10.7202/037217ar

\section{Résumé de l'article}

Le québécisme nordicité: de Ia néologie à Ia lexicalisation - Nordicité - état de " Nord » - reçoit ses premiers contextes en français en 1965, et, en anglais, quelques années après. Plus tard, le mot apparaît dans des titres. Au cours de la décennie 1980, les grands dictionnaires Larousse et Robert le consignent. Servant de tronc, il donne naissance à un corpus d'une soixantaine d'entrées en langue française. Le nordic nord-américain conduira à nordicity. Des adaptations du mot existent en quelques autres langues. L'étude de cette évolution vocabulairique apporte quelques lumières sur l'histoire des racines nord|north, le sens Scandinave du tronc nordique, la création lexicale elle-même, les avantages et inconvénients de toute entité, le cheminement des signifiants auprès des usagers, les processus de lexicalisation, l'apport québécois aux parlers hors-Québec ainsi que la double destinée d'un mot en langue courante et d'un terme en langue de spécialités.
Tous droits réservés @ C TTR: traduction, terminologie, rédaction — Les auteurs, 1995
Ce document est protégé par la loi sur le droit d'auteur. L'utilisation des services d'Érudit (y compris la reproduction) est assujettie à sa politique d'utilisation que vous pouvez consulter en ligne.

https://apropos.erudit.org/fr/usagers/politique-dutilisation/ 


\section{Le québécisme nordicité: de la néologie à la lexicalisation}

\section{Louis-Edmond Hamelin}

Le néologisme de nordicité est l'objet d'un déploiement manifeste dont veut témoigner cette monographie à la fois lexicologique et terminologique.

\section{I. Écologie d'un mot à créer}

Nordicité constitue un anneau dans une chaîne de dérivation. En amont se trouvent nordique, et en amont de ce dernier, nord. En aval, apparaissent d'autres entités dont et nordiciste et nordicitude.

Nord

Même si l'étymologie et la datation sont mal fixées, il s'agit d'un signe ancien qui voyage beaucoup au cours des siècles. D'après une vue planétaire et millénaire des choses, il s'étend suivant deux principaux axes déphasés et perpendiculaires: l'un, dans le sens des longitudes, relie dès l'Antiquité les mondes méditerranéens et scandinaves (et viceversa); cette migration peut expliquer que les deux principales langues qui utilisent la lexie nord sont germaniques et romanes.

À partir de la Renaissance, va se développer le second axe du langage nord, celui d'une direction est-ouest quart au nord, s'étirant à la fois de l'Europe vers l'Asie et de l'Europe vers l'Amérique. C'est ainsi qu'un Nordvik norvégien atteint la Sibérie arctique et qu'un Nordseta viking débarque au Labrador. Cette migration, d'abord côtière, pénétre profondément à l'intérieur des continents, migration qui amène 
un Northwest britannique au lointain Mackenzie(ou Deh Cho, en langue autochtone).

Ce double itinéraire géographique a ses conséquences au plan du langage. D'une part, la dimension translatitudinale aide à comprendre l'esprit "sudiste» et "pays tempérés» des aventures polaires des NonAutochtones. D'autre part, même si la substance nordique de la seconde aventure est plus élevée que celle de la première, le vocabulaire restera comme limité par le sens imprécis et faiblement nordiciste des mots européens de la première phase. Par le fait même, il deviendra difficile de décrire des régions canadiennes et russes possédant des états boréaux aigus et zonés en utilisant simplement des termes et des concepts établis pour rendre compte d'une polaricité basse et moins hétérogène. Le sens des mots froids européens devient inapproprié dans les pays vraiment polaires. Les outils de la pensée atteignent des régions éloignées pour lesquelles il n'avaient pas été conçus. Toute cette inadaptation conduit à de mauvais entendements des choses arctiques.

Le mot nord lui-même pose le problème fondamental de l'objectivité ou de la subjectivité de sa représentation. Pourrait-on prétendre que l'image du Nord ne demeure qu'une vue des pays moins sévères? La tendance de ceux-ci d'élaborer des normes applicables à la terre entière jouerait-elle contre l'élaboration d'une conception pertinente de la zone froide? Sans doute que cette vision coloniale ait été largement véhiculée, même par les savants. Mais la reconsidération de l'entité «nord» - sourçant d'une première nordologie ou étude du Nord - fera prendre une distance notionnelle par rapport aux concepts antérieurs ancrés dans les zones tempérées. En conséquence, nous abordons le concept zonal d'après une analyse polaire autonome. Pendant de longs mois, la situation des pays des hautes latitudes tient à un angle désavantageux des rayons solaires sur les surfaces inclinées. Cette seule constatation montre les limites des recherches qui, prioritairement, fonderaient la personnalité du Nord sur les caractèresdu Sud. La nouvelle notion nordique veut directement rejoindre le propre, l'être, l'existentiel dé la zone froide. Des référents caractéristiques sont la lumière ou l'obscurité dielles, le pergélisol continu ou non, la durée de la couverture glacielle (glaces flottantes), l'autochtonité ainsi que par le «State of Mind» des immigrants. Heureusement, la plupart de ces traits sont directement saisissables par un indice spécifique. Cette 
démarche récente appréhende donc le Nord comme une réalité première, non comme une réalité subordonnée à toute autre.

\section{Nordique}

Le mot exprime la première génération de l'évolution lexicale de nord. Le développement sémantique de l'adjectif et du substantif a connu trois principales périodes en un peu plus d'un siècle.

A. En français, le mot est relativement récent. Littré (1873) ne l'emploie pas alors que Le Robert le fait apparaître au dernier quart du XIX siècle. Il est essentiel de retenir que les lexicographes lui font signifier "uniquement scandinave» (en référence aux péninsules sises au nord de l'Europe). Nordique montre une fréquence faible, des dictionnaires, même récents, ne lui consacrant pas toujours d'entrée autonome. De son côté, nordic se dit : «Of the tall blond dolichocephalic people found esp. in Scandinavia», d'après l'Oxford Dictionary.

B. Nous sommes déçu de n'avoir pas accès à une plus grande capacité sémantique et spatiale du mot. Aussi, durant la décennie cinquante, nous est-il venu à l'idée de faire deux propositions: a) élargir l'application de nordique à tous les pays froids de latitude, conformément à l'amplitude de nord lui-même; par cette extension, le nouveau sens deviendrait disponible au CanadalQuébec; b) lui donner un contenu thématique qui ne se limiterait pas à la linguistique norse et à des ethnies européennes; ainsi, l'entité se dirait de tous les aspects pertinents de la zone froide de latitude. Peu d'années plus tard, c'est cette dimension élargie que véhicule mon mémoire Pour un centre nordique, document déposé à l'Université Laval et au Gouvernement du Québec. Mais la nouvelle acception demeure toujours hors-la-loi; d'après les dictionnaires, la publicité de l'organisme québécois de recherches se trouve inexacte, voire trompeuse. Le malaise va durer jusqu'à la lexicalisation du nouveau sens de nordique, notamment par les grands dictionnaires parisiens, au début des années 80 .

C. Pendant les vingt ans de purgatoire, la signification «nord du monde» fait son chemin, heureusement sans remplacercelle de «nord-européen»; la comparaison des rubriques dans les Larousse (1948 et 1993) démontre cette évolution dans les significations. Au cours des années 
soixante, le nouvel usage commence à se répandre; les fonctions se multiplient. L'expression Monde nordique entre dans des productions médiatiques, dans l'organigramme de plans de cours, dans des ouvrages de prestige, dans des atlas... La France s'y met; la Documentation Photographique de Paris écrit en 1982: «Nous donnons aux régions nordiques le sens, maintenant largement accepté en Amérique du Nord, de pays froids de l'ensemble du monde circumpolaire boréal».

Bref, dans l'Hexagone, nordique référant déjà à des «faits scandinaves» prend un sens linguistique vers 1875 . Au Québec, au cours de la décennie cinquante, le territoire du terme devient le «nord du monde». Vingt-cinq ans après, des dictionnaires de langue lexicalisent cette seconde définition.

\section{L'arrivée de nordicité}

Le néologisme de nordicité dont les premières citations datent de 1965 tient compte du développement sémantique des deux racines: nord et nordique. Il veut exprimer à la fois la dimension universelle de nord (contrairement à nordique rivé à l'Europe) de même qu'un contenu apte à rejoindre toutes les situations thématiques circumpolaires, ce que ne faisait aucun des deux troncs. Cette double conception est alors toute nouvelle en français et dans d'autres langues.

\section{Notions}

Nordicité, substantif féminin, peut se définir ainsi: État, niveau, fait et conscience de toutes choses concernant les hautes latitudes boréales.

En 1965, deux contextes de fondation apparaissent à la fois au Canada et en France; voici celui d'un périodique français. Quatre autres citations suivront.

1. «Réunir par des traits les lieux de l'hémisphère boréal qui possèdent le même nombre d'unités nordiques ou la même nordicité». LouisEdmond Hamelin, Leçons télévisées... Revue de Géographie Alpine, Grenoble, 53, 1965, p. 667. (L'énoncé renvoie à la nordicité géographique, voir plus loin.) 
2. «Est-il manière plus heureuse d'introduire une géographie du Canada que de parler de sa nordicité». Paul Claval, Le Monde, Paris, 22 novembre 1969 , p. VI.

3. «Northwest Canada possesses the steepest gradient of nordicity as, within a few hundred miles, the forest of Lower Mackenzie give way to permanent polar pack». Canada. A Geographical Perspective. Toronto, Wiley, 1973, p. 35. (Traduction par M.C. Storrie et C.I. Jackson).

4. «Un matin à Yellowknife, il [L.-E. H.] crée le mot nordicité pour désigner une réalité géographique, un territoire méprisé par les initiatives, les comportements...». Conrad Bernier, La Presse, Montréal, 22 mars 1976, p. D-7.

5. "The notion of nordicity captures the essence of a distinctive Canadian identity that should be nurtured in external as well as internal affairs»r. F. Griffiths, A Northern Foreign Policy, Toronto, Wellesley Papers, 7, 1979, p. 20.

\section{Réceptivité internationale du néologisme}

Les contingences extérieures affectent la destinée du canadianisme. Les contrées finno-scandinaves peuvent craindre que la nouvelle dimension circumterrestre de nordicité les affecte en enlevant l'exclusivité notionnelle de leur nordique historique et en relativisant leur niveau seulement moyen de «Nord» par rapport aux niveaux plus sévères du Canada et de la Russie. Pour leur part, des Britanniques hésitent devant un signe dont la charge ethnique peut réveiller de mauvais souvenirs (supériorité hitlérienne d'une race «nordique»). À l'occasion, l'ex-URSS traduit le mot (Izvestiya, Moscou, 1972) mais la Guerre Froide n'est pas favorable à l'échange vocabulairique entre le Canada et l'empire soviétique. Quant à l'Amérique anglo-saxonne, elle tolère à la fois nordicity, formé à partir du -dic de nordic, de même que The Québec Nordiques. Dans les pays chauds, le terme est inutile si ce n'est pour parler des territoires climatiquement opposés; néanmoins, des chercheurs utilisent telle quelle la forme québécoise. En France, nordicité apparaît à diverses tribunes: Monde, posters de conférence à Toulouse et à 
Grenoble, mention dans des manuels publiés par de grands éditeurs (Hachette, Masson), entrée dans des dictionnaires spécialisés, article sur la situation comparée des pays circumpolaires, index de la Banque des mots, dictionnaires de langue. Même si, à l'intérieur de l'Hexagone, les Français n'ont pas besoin de l'entité, ils l'accueillent quand même.

Nous ignorons presque tout de l'emploi de nordicité et nordicity dans les langues autres que le français et l'anglais. Cependant, des mots probablement peu utilisés existent: nordlichkeit en allemand, nordiskhet en danois, nordicitad en espagnol, nordicità en italien, norsket en norvégien, puis en cyrillique russe. Dans la traduction de ses résumés d'article, la revue internationale Forces de Montréal a eu la coquetterie de le faire apparaître en arabe, japonais et chinois.

6. "The system of assigning polar values or VAPOS in ten scales of nordicity has the merit of blending the objective and the subjective». A. Skenpiel et N.A. Easton, New Bearings on Northern Scholarship, The Northern Review, Whitehorse, 1, 1988, p. 18.

7. "Con l'expressione Grande Nord, s'intende una zona di nordicità elevata, ma non massima...»Bolletino Della Società Geografica Italiana, Rome, 10, 3, 1974, p. 276.

\section{Attitudes du public}

L'accueil ou le refus des utilisateurs potentiels module largement la carrière de nordicité. Les attitudes sont multiples. Objectivement, l'absence antérieure de moyen pour exprimer le degré de "nord", englober l'ensemble thématique circumpolaire ainsi que fixer une limite méridionale à la zone froide joue en faveur de l'acceptation du néologisme. Des usagers semblent même assoiffés du nouveau technolecte.

Par contre, les spécificités idéelles du mot n'offrent qu'un intérêt limité aux chercheurs sectorialistes, factuels et non régiologues. De plus, les exigences appliquées de la nordicité mentale rebutent les savants qui ne tiennent pas à s'engager en faveur des causes du Nord. Par ses dimensions globales et morales, le message de la nordicité ne 
sourit donc pas également à chacun. Nordicité ne posséderait-il pas quelques traits d'un psycholecte?

Subjectivement, existent aussi toute une gamme de positions qui favorisent ou non la circulation d'un néologisme. Par exemple, il se trouve des «retardataires» (Wijnands), ces locuteurs qui, techniquement, attendent la lexicalisation définitive d'une entité avant de commencer à l'utiliser. Or, ils peuvent toujours juger insatisfaisantes les attestations courantes et, partant, persister dans leur abstention. Par comparaison, au Québec, un ostracisme prolongé a touché le mot rang, au sens de «rang d'habitat».

Au total, la carrière d'un nouveau venu est imprévisible, mais, après trente ans, on pourrait reconnaître un certain succès à nordicité.

\section{Champs d'application}

Notre objectif concerne l'histoire d'une entité lexicale qui, destinée à une langue de spécialité, connaît aussi un cheminement en langue courante. Ici, il ne s'agit pas d'argumenter sur la matière nordiciste ellemême. Le traitement est donc terminologique, non nordologique. Voici six domaines d'emplois.

\section{La nordicité géographique}

Le déterminatif renvoyant à une géographie d'amplitude parfois désignée totale ou globale s'accorde avec la capacité du substantif. $\mathrm{Ce}$ type de nordicité «matérielle» trouve sa définition dans l'utilisation des facteurs identifiés dans la géographie théorique des hautes latitudes.

Un indice identifiant les valeurs polaires permet d'évaluer l'identité de tous lieux ou faits de la zone froide de l'hémisphère boréal. En outre, il fournit le moyen de connaître les facteurs qui contribuent le plus ou le moins à la polaricité moyenne. La saisie d'un tel différentiel conduit à d'utiles comparaisons intranordiques, par exemple, entre la Sibérie et les Territoires du Nord-Ouest au Canada. L'immense monde polaire forme une entité territoriale, non au sens que tous les éléments y sont semblables, mais à celui que les gens et les choses qui s'y trouvent sont en liaison, d'une façon harmonieuse ou non. 
L'évolution de la pensée et le mot nordicité font donc passer des anciennes notions sectorielles d' "arctique thermique» $\left(10^{\circ} \mathrm{C}\right.$ en juillet) et de tree line subarctique à une notion d'amplitude, celle d'une «région plurifactorielle». Une dimension transdisciplinaire féconde donc la sémantique du néologisme.

8. «Dans l'Arctique, ce qui reste inchangé, c'est notre [réfère aux Inuit] enracinement dans notre terre, notre climat, notre nordicité». Mary Simon, Biennale des Villes internationales d'hiver, Montréal, Plénière, 18 janvier 1992.

\section{La nordicité mentale}

Les acceptions autres que géographiques se retrouvent dans le titre même de notre ouvrage de 1975 où, à tout risque, apparaît le néologisme de nordicité, et non le mot usuel, plus "comestible», de nord. Par le suffixe -ité, nous invitions spécifiquement le lecteur à privilégier l'état et l'évaluation des choses plutôt qu'à recevoir des informations non engageantes. Cette idée que le Nord est dans la tête, dans l'être, et non seulement dans la nature, nous était venue en écoutant les conférences de l'explorateur V. Stefansson à McGill à la fin des années quarante.

9. "Sweden adopted a self-image of what one could call nordicity». Sverker Sörlin, Nordic Identity: Sweden and Canada in a comparaison perspective. Abstracts. Canada and the Nordic Countries. Lund, Suède, The Nordic Association of Canadian Studies, 1987, p. 76.

10. «Most Canadians have yet to develop a true mental nordicity». Lise Bissonnette in Globe and Mail, Toronto, 23 avril 1988, p. D-8.

\section{Autres nordicités thématiques}

À l'occasion, la notion nouvelle a été utilisée en biologie, glaciologie, ingénierie, sciences naturelles, démographie de même que dans l'administration territoriale. Certains chercheurs viennent d'agrandir le domaine politique de nordicité en appliquant la notion à des interactions entre les peuples du Nord. 
Le terme entre aussi dans les domaines techniques.

11. «... en attendant que les impératifs du marché ou qu'une législation gouvernementale n'oblige les fabricants d'automobile à la nordicité de leurs véhicules vendus au Canada». R. Fleury, Le Soleil, Québec, 16 avril 1977, p. E-10.

12. «Les balanes, petits crustacés, représentent-ils un cas d'ajustement des espèces à la nordicité?» M. Jugler, Contact, Québec, Un. Laval, I, 2,1987 , p. 35 .

\section{Les nordicités territoriales}

Étant donné que l'objet - le Nord - constitue une étendue et que la géographie est l'une des disciplines de son appréhension, la nordicité comportera évidemment un référent spatial. D'abord, une certaine littérature sera consacrée aux limites mêmes du Monde nordique. Une autre va considérer les subdivisions physico-humaines de cet univers rigoureux. On individualise un Moyen Nord: «zone du monde circumboréal caractériséepar un niveau intermédiaire de nordicité». Des auteurs parlent d'espaces, comme nordicité internationale, définis par d'autres échelles et structures.

13. «Nordicity is a measure of the degree of northernness of a place. It provides a quantitative definition of a southern boundary of the North". R.B. Bone, The Geography of Canadian North. Toronto, Oxford, 1992, p. 249.

En outre, l'indice s'est appliquée aux deux cônes circumpolaires de la planète, le Monde nordique, bien sûr, mais aussi l'Antarctique.

Au Canada, les emplois de nordique (au sens «tour de la planète) et de nordicité, d'abord restreints au vrai Nord défini par l'indice, gagnent du terrain vers le sud. Les deux mots en viennent même à exprimer la façade méridionale du pays. En ce faisant, les usagers dépassent la pensée du néologue premier! Évidemment, c'est la saison d'hiver qui attire ces termes dans le Sud canadien et le Nord des États-Unis. On parle de nordicité temporaire ou hivernale. En fait, les rapprochements entre le concept de région et de saison froide vont de 
soi. En 1990, une Foire internationale du livre annonce L'homme et l'hiver au Canada [méridional] du Français Pierre Deffontaines (Paris, 1957) comme un «aspect de la nordicité». Ainsi, par l'application du vocabulaire nordique aux situations hivernales du Canada de base, c'est tout le territoire national qui est en train d'adopter le langage nordiciste. On décrit la saison nivale du Sud canadien en s'appropriant le vocabulaire strict du Nord. Les façades méridionales des Pays Finnoscandinaves font de même depuis longtemps.

\section{En langue courante}

Nordicité, né comme terme d'une langue de spécialité, est devenu un simple mot dans la langue quotidienne. C'est justement la pénétration de l'entité dans le parler commun qui en a assuré l'extension. Tout en demeurant employée à un certain niveau de spécialisation (cas de la nordicité géographique), elle se retrouve dans les langages des sports, de la construction, du tourisme, transport et commerce. Les grands journaux: Le Monde, Paris, The Globe and Mail, Toronto et La Presse, Montréal, s'en servent à l'occasion.

14. «Montréal ne sait plus affronter l'hiver [retard dans le déneigement]. Que notre ville retrouve et assume sa nordicité». $P$. Cayouette, Le Devoir, 17 février 1993.

15. «La nordicité québécoise s'est gravée dans sa sensibilité déjà éveillée à cette appartenance aux éléments septentrionaux: vent, froid, eaux impétueuses, horizon sans limite». Thérèse Dumesnil, Suite Nordique. Baie de James, Perspectives, Montréal, 27 octobre 1979, p. 12.

La lexicalisation dictionnairique: Grand Robert, Petit Larousse et les dictionnaires du Québec, comme le Dictionnaire québécois d'aujourd'hui et le Dictionnaire du français plus agrandissent à leur tour la plage d'expression de nordicité.

16. «NORDICITÉ. Caractères relatifs aux régions et aux habitants situés dans toute la partie septentrionale de l'hémisphère boréal». Dictionnaires Le Robert, Grand Robert, Paris, tome VI, 1985, p. 803. 
La vie artistique et littéraire accueille aussi le nouveau signe, comme en font foi les contextes suivants:

17. «Faire de la peinture jusqu'à la nordicité». René Derouin, Cahier d'exposition, Trois-Rivières, 1979, p. 43 (représentation de la Suite nordique du Nouveau-Québec).

18. "That elusiveness is the core of the novel's nordicity". Ted Blodgett, Functions of the North in Gabrielle Roy's La Montagne Secrète, Abstracts. Canada and the Nordic Countries. Lund, Suède, The Nordic Association of Canadian Studies, 1987, p. 7.

19. «Au diapason de la nordicité de leur pays, les compositeurs canadiens réussissent à s'imposery. K. Bagnell, [La musique], La Revue de l'Impériale, Toronto, été 1991, p. 3.

20. «Monsieur Roland Arpin à l'occasion de l'exposition sur la nordicité au Musée de la Civilisation [à Québec]: un million de visiteurs». $L a$ Presse, Montréal, 29 mars 1990.

21. La nordicité. Sketch par Pierre Turgeon et Chantal Strivastava, Radio-Canada, CBV, Québec, 23 janvier 1990, 6 minutes.

22. «Tous ces articles sur l'hiver illustrent que la langue française sait rendre avec différentes tonalités l'atmosphère de la nordicité». Yvon Bellemare, Québec français, Québec, 88, 1993, p. 83.

\section{Famille de mots}

Nordicité ne demeure pas une entité orpheline dans les langages courant et scientifique. Petit à petit, des auteurs l'utilisent comme tronc pour y greffer préfixes, suffixes, formants composés et associés. La nordonymie (étude du vocabulaire thématique et régional concernant les pays froids des latitudes boréales) s'est enrichie. En conséquence, par rapport à la génération précédente, on peut exprimer et comprendre davantage les situations nordiques, et le faire avec plus de nuances. 
Tableau 1 - Provignement de nordicité (relevé factuel)

\author{
adaptation \\ nordicity \\ (Autres langues, voir le texte.) \\ composition \\ anordicité \\ prénordicité \\ paléonordicité \\ néonordicité \\ nordicisant (nordicant) \\ nordicisé \\ nordiciste \\ nordicitude
}

association d'entités (quatre groupes)

référent territorial

nordicité canadienne

nordicité internationale

nordicité intranationale

nordicité locale

nordicité nationale

nordicité océanique

nordicité ponctuelle

nordicité provinciale

nordicité québécoise

nordicité régionale

nordicité zonale

référent thématique

nordicité appliquée

nordicité biogéographique

nordicité circumpolaire

nordicité climatique

nordicité comparée

nordicité développementale référent thématique (suite) nordicité géographique nordicité géopolitique nordicité globale nordicité historique nordicité hivernale nordicité naturelle nordicité quantitative nordicité sportive nordicité technique

référent temporel nordicité actuelle et subactuelle nordicité ancienne nordicité évolutive nordicité momentanée nordicité prospective nordicité saisonnière

référent mental nordicité ajustée nordicité appropriée nordicité déductive nordicité déficiente nordicité d'usage nordicité empirique nordicité fausse nordicité imaginaire nordicité imitative nordicité improvisée nordicité instinctive nordicité insuffisante nordicité intérieure nordicité mentale nordicité normative nordicité psychologique nordicité rationnelle nordicité répulsive nordicité théorique 
23. "Les mots-valise sont issus d'une réduction d'une suite de mots à un seul; on conserve la partie initiale du premier mot et la partie finale $\mathrm{du}$ dernier; exemple nordicitude: nordic[ité] + [sol]itude]". J.-C. Boulanger et Michèle Rivard, in Néologie en marche, Québec, OLF, série $b, 1,1976, \mathrm{p}$. XV.

La famille nordique complète rassemble trois champs étymologiques. Un premier utilise le mot nordicité lui-même et comprend plusieurs dizaines d'entrées (tableau 1). Le deuxième réfère à d'autres expressions de la famille "nord" parfois présentes dans les dictionnaires de langue, ainsi, Grand Nord, Moyen Nord, Nord, Norden, nordique, nordisme, nordiste, nordologie, Northnam... Enfin, sont aussi utilisés des mots du vocabulaire polaire çonstruit à partir de racines diverses dont arctique, cryo et géli.

\section{Conclusion}

Le terme et le mot nordicité qui se rapportent aux pays froids de latitude ont acquis un statut d'existence et fait naître toute une famille d'entités lexicales. Aussi trouvons-nous de nombreux contextes d'emploi dans l'une ou l'autre, et parfois dans l'une et l'autre, des catégories classiques de lexicalisation: dictionnaires de langue, documents légaux, ouvrages de prestige, banques de données, glossaires spécialisés, livres et articles, télémissions, littérature, œuvres d'art, enfin, pénétrations dans d'autres langues que le français et l'anglais. Même si la lexie demeure disponible pour de nouveaux développements, il ne s'agit plus d'un néologisme.

\section{Louis-Edmond Hamelin: 1244 Albert-Lozeau, Sillery, Québec G1T 1 H4}

\section{Références}

(autres que les références portées dans le texte)

BOLDUC, André, dir. (1990). «L'hiver au Québec», numéro spécial, Forces, Montréal, 88, 80 p. (hiver et nordicité). 
CABOURET, M. (1989). «Propositions d'une délimitation des zones nordiques en Fenno-Scandie», Hommes et Terres du Nord, Lille, 3, pp. 154-160.

CAILlEUX, A. et A.A. VELICHKO (1972). «Un indice circumpolaire" (en russe), Izvestiya Akademii Nauk, SSSR, Seriya geografischevskaya, Moscou, 3, pp. 121-133 (adaptation à l'Antarctique).

HAMELIN, Louis-Edmond (1975). Nordicité canadienne. Montréal, HMH, 458 p.

GRAHAM, Amanda (1990). «Indexing the Canadian North: Broadening the Definition», The Northern Review, Whitehorse, 6, pp. 21-37.

WIJNANDS, Paul (1985). «Pour une redéfinition du néologisme lexicographique», La banque des mots, Paris, 29, pp. 13-34.

WONDERS, W.C., dir. (1971). «A Circumpolar Index», Canada's Changing North. TorontolMontréal, MCS, The Carleton Library, 55, pp. 7-21.

RÉSUMÉ: Le québécisme nordicité: de la néologie à la lexicalisation - Nordicité - état de «Nord» - reçoit ses premiers contextes en français en 1965, et, en anglais, quelques années après. Plus tard, le mot apparaît dans des titres. Au cours de la décennie 1980, les grands dictionnaires Larousse et Robert le consignent. Servant de tronc, il donne naissance à un corpus d'une soixantaine d'entrées en langue française. Le nordic nord-américain conduira à nordicity. Des adaptations du mot existent en quelques autres langues. L'étude de cette évolution vocabulairique apporte quelques lumières sur l'histoire des racines nord Inorth, le sens scandinave du tronc nordique, la création lexicale elle-même, les avantages et inconvénients de toute entité, le cheminement des signifiants auprès des usagers, les processus de lexicalisation, l'apport québécois aux parlers hors-Québec ainsi que la double destinée d'un mot en langue courante et d'un terme en langue de spécialités. 


\begin{abstract}
The Québec Term "Nordicité»: From Neologism to Lexical Derivation - Nordicité - the state of «Nord» - was coined in French in 1965, used soon after in English, and later appears in titles. During the 1980's, it is included in important French dictionaries, such as Larousse and Robert, giving rise to a corpus of some 60 derivations. Nordicity is a North-American derivation from Nordic. Adaptations of this term also exist in some other languages. The study of its evolution can shed some light on the history of the root words nord lnorth, the Scandinavian meaning of Nordic, lexical creation itself, the advantages and disadvantages of any lexical unit, the acceptance of new words, the process of lexical derivation, Québec's contribution to language usage outside Québec, the double role of words (common language) and of terms (specialized language).
\end{abstract}

